Abstract

\title{
Artemia salina Contamination with Glyphosate and Treatment with Isotherapic
}

\author{
Mirian Yaeko Dias de Oliveira Nagai*, Andreia Adelaide Pinto, Ednar do Nascimento Coimbra \\ Melo, Suham Nowrooz, Ivana Suffredini, Maria Martha Bernardi, Leoni Villano Bonamin
}

Universidade Paulista, São Paulo, Brazil

*mynagai@yahoo.com.br - https://orcid.org/0000-0001-8410-0794

Introduction Artemia salina, a micro-crustacean of the order Anostraca, is one of the most used ecotoxicity bioindicators. Glyphosate is an herbicide widely used in the world, whose toxicity is still controversial, although many evidences point to endocrine disruption effects. Objective Since in homeopathic practice intoxications are often treated by isotherapy, our objective was to verify the possible protective effect of glyphosate isotherapics, in different potencies, when added to sea water in which Artemia salina were exposed to $0.02 \%$ glyphosate (corresponding to CL10). Behavioral and morphological parameters were evaluated. Methods Artemia salina cysts were kept in culture bottles containing artificial sea water, following standard technique of oxygenation, luminosity and temperature to promote hatching within 48 hours. The isotherapic preparations were inserted in each bottle in a $10 \%$ of the total water volume. The obtained nauplii and water samples were frozen to further glyphosate dosage. Part of the nauplii was distributed in transparent tubes, being 10 nauplii per tube and 6 tubes per group, for behavioral analysis, and part were collected for a detailed morphological analysis, under an optical microscope. Results and Discussion About 80 to 270 nauplii were analyzed per group. The data of healthy/defected nauplii ratio presented statistical significance $\mathrm{p}<0.04$ (ANOVA) in Glyphosate $6 \mathrm{CH}$ and Glyphosate $30 \mathrm{CH}$ groups, in relation to controls. Glyphosate $6 \mathrm{CH}$ also increased ( $\mathrm{p}=0.01$ ANOVA) the frequency of nauplii swimming on the surface water column, what means higher vitality. Glyphosate $6 \mathrm{CH}$ and Glyphosate $200 \mathrm{CH}$ reduced the frequency of cyst hatching ( $\mathrm{p}<0.05$, ANOVA). These preliminary data suggest a kind of "selective" effect of isotherapics, in which few but resistant nauplii were born. Conclusion We concluded that the use of isotherapics might be an interesting tool to reduce the environmental impact of the indiscriminate use of glyphosate.

Keywords: Artemia salina, glyphosate, isotherapic, homeopathy, eco-toxicity

(C) International Journal of High Dilution Research.

Not for commercial purposes. 